\title{
Schrödinger Dynamics and Physical Folia of Infinite Mean-Field Quantum Systems
}

\section{T. Unnerstall}

Institut für Theoretische Physik der Universität Tübingen, Auf der Morgenstelle 14, D-7400 Tübingen, Federal Republic of Germany

\begin{abstract}
This work deals with the general (i.e. non-equilibrium) Schrödinger dynamics of infinite mean-field quantum systems. It is shown how this dynamics is related to the Hamiltonian flow $\varphi_{t}^{Q}$ on the "classical phase-space" $E \subseteq \mathbb{R}^{L}$ recently defined by Bona [10] to describe the time evolution of classical (macroscopic) observables of the system. These connections allow us to clarify the structure of the set of all physical folia, a notion introduced by Sewell for the dynamical description of infinite systems in cases where this description is representation-dependent. They also yield a result showing that the Heisenberg picture is the more general approach to such descriptions in the sense that there are more representations in which a Heisenberg dynamics can be defined than ones which allow for the definition of a Schrödinger dynamics. Finally, our theory makes it possible to construct many explicit examples of physical folia; in this connection it is shown that there can be overcountably many inequivalent representations with the same macroscopic dynamical structure.
\end{abstract}

\section{Introduction}

Recently, great progress has been made concerning the rigorous formulation of the general, i.e. non-equilibrium dynamics of infinite quantum (lattice) systems with a mean-field interaction.

Such models were first considered by Hepp and Lieb [1], who showed that they can be successfully applied to problems in superconductivity and laser-physics. One interesting feature of infinite mean-field systems is that, due to the extremely long range of the interaction, the Heisenberg dynamics cannot be defined as a *-automorphism group of the $C^{*}$-algebra $\mathscr{A}$ of quasilocal observables [2]; or, expressed in the Schrödinger picture, that the "set of physical states" [3] is not all of $S(\mathscr{A})$, the state space of $\mathscr{A}$. While the dynamical description of the system in thermal equilibrium -i.e. in the GNS-representation of KMS- or limiting Gibbs-states - was discussed extensively in the literature [4], the concepts necessary to deal with the general case developed more slowly.

Sewell [3] worked in the Schrödinger picture and used the notion of a "folium" 
in $S(\mathscr{A})$ introduced by Haag et al. [5]; a folium $F \subseteq S(\mathscr{A})$ is a convex, normclosed set of states $\omega$ on $\mathscr{A}$, which is invariant under "quasilocal perturbations" in the sense that

$$
\omega \in F \Rightarrow \omega_{c}:=\frac{\omega\left(c^{*} \cdot c\right)}{\omega\left(c^{*} c\right)} \in F \forall c \in \mathscr{A} \quad \text { with } \quad \omega\left(c^{*} c\right) \neq 0 .
$$

Folia arise naturally as the set of $\pi$-normal states of any representation $\pi$ of $\mathscr{A}$; more precisely, there is a one-to-one correspondence of folia in $S(\mathscr{A})$ and quasiequivalence classes of representations of $\mathscr{A}$. Thus, if $\mathscr{M}_{\pi}=\pi(\mathscr{A})^{\prime \prime}$ denotes the von Neumann algebra belonging to $\pi$, the folium $F_{\pi}$ belonging to $\pi$ is $F_{\pi}=\left(\mathscr{M}_{\pi}\right)_{*,+, 1}$. Sewell defined as a "physical folium" of the system a folium on which an affine, normpreserving group of transformations $v_{t}: F \rightarrow F$ acts, which is in a natural sense the limit of the local time evolutions and which can thus be regarded as the Schrödinger dynamics of the infinite system. Consequently physical folia in $S(\mathscr{A})$ constitute possible dynamical descriptions in the Schrödinger picture of the quantum system described by $\mathscr{A}$.

This dynamical theory can be completed due to the fact that, in $\pi_{F}$, the representation belonging to $F$, one can also define a Heisenberg dynamics [6] as a group of $W^{*}$-automorphisms $\tau_{t}: \pi_{F}(\mathscr{A})^{\prime \prime} \rightarrow \pi_{F}(\mathscr{A})^{\prime \prime}$, which is the dual of $v_{t}$. The Heisenberg dynamics was analysed further by Morchio and Strocchi [7], who emphasized the importance of operators in the center of the von Neumann algebra

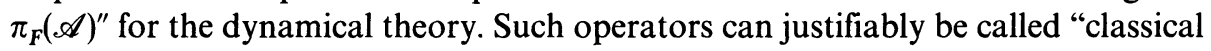
observables" [8], and thus play a crucial role from a fundamental point of view [9].

Decisive progress in this line of thought was recently made in the impressive work of Bona $[10,11]$, who worked in the Heisenberg picture and proved the existence of the limiting Heisenberg dynamics in a certain maximal representation $\pi_{\mathscr{G}}$ (with folium $F_{\mathscr{G}}$ ) for a large class of mean-field models. He was able to clarify completely the rôle of the classical observables in the dynamics of the infinite quantum system and described their time evolution by means of a "classical phasespace" $E \subseteq \mathbb{R}^{L}$ and flow $\varphi_{t}^{Q}$ on $E$. Each point in this phasespace $E$ corresponds to certain values of the classical observables, hence to a set of macroscopic parameters of the system: its "classical state." The flow $\varphi_{t}^{Q}$ being generated by a Hamiltonian function $Q$, Bona thus realized rigorously old ideas of Hepp and Lieb [1]. The structures developed by Bona can in fact be generalized by associating

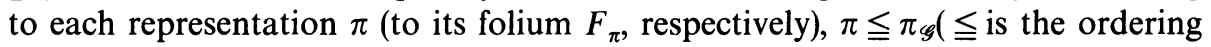
of quasi-equivalence classes of representations), a classical space $E_{\pi} \subseteq E$ in a natural way [12]; for the limiting Heisenberg dynamics to exist in $\pi$ it then suffices that $E_{\pi}$ be invariant under $\varphi_{t}^{Q}$.

The purpose of the present work is to investigate the Schrödinger dynamics of the class of models considered by Bona. Working in the framework of the folium $F^{p} \subseteq F_{\mathscr{G}}$ generated by the permutation-invariant states $S^{p}(\mathscr{A})$, which is slightly smaller than $F_{\mathscr{G}}$, we are pursuing two goals.

The first goal is a description of the Schrödinger dynamics $v_{t}^{Q}: F^{p} \rightarrow F^{p}$ which is as explicit as possible. This is done with help of the flow $\varphi_{t}^{Q}$ : we show how the full quantum state evolution of $\omega \in F^{p}$ is constrained (or, in the case $\omega \in S^{p}(\mathscr{A})$, even fully determined) by the time evolution of the macroscopic parameters which a(n) 
(ensemble of) system(s) described by $\omega$ can assume. Thus we establish rigorously the connection between the microscopic, quantum level of state evolution $\left(v_{t}^{Q}\right)$ and the macroscopic, classical level of state evolution $\left(\varphi_{t}^{Q}\right)$.

Noting first that the considered class of models, and more explicitly the folium $F^{p}$, is a concrete example of the notion of a physical folium considered abstractly in [3] — this has already been pointed out in [13] — the second goal of our work is to elaborate this notion further by giving many more examples of physical folia, and by analysing the structure of the set of all physical folia (in the framework of $\left.F^{p}\right), \mathbb{F}_{\text {phys. }}$. In particular, we show how $\mathbb{F}_{\text {phys }}$ is naturally classified in terms of the "sub-phasespaces" $B \subseteq E$, i.e. the closed, $\varphi_{t}^{Q}$-invariant subsets of $E$. These correspond physically to certain ranges of the macroscopic parameters to which the system may be confined due to external circumstances such as temperature (compare [14]). For each such physical situation, then, our theory

- defines the set of all dynamical descriptions (= physical folia) of the system which are at one's disposal in this situation;

- gives a method to construct such descriptions explicitly.

These insights can be used, for example, to construct descriptions in which a Hamiltonian generating the dynamics can be defined [15].

Having thus given an overview of the physical concepts involved, we now briefly sketch the technical procedure of the paper. After defining the class of models under consideration and introducing the Schrödinger dynamics $v_{t}^{Q}: F^{p} \rightarrow F^{p}$ in Sect. II, we first look at the general structure of $\mathbb{F}_{\text {phys }}$ (Sect. III). We show that the time development of subfolia of $F^{p}$ is reflected by the classical flow $\varphi_{t}^{Q}$ in a "coarse grained" way. This is then used to introduce the natural classification of $\mathbb{F}_{\text {phys }}$ (in terms of the sub-phasespaces $B \cong E$ ) referred to above. We also show in this connection that the relation between $v_{t}^{Q}$ and $\varphi_{t}^{Q}$ is "too coarse" to allow an identification of all physical folia by means of $\varphi_{t}^{Q}$, which contrasts to some extent the situation in the Heisenberg picture. An interesting consequence of this state of affairs is that there are many representations $\pi$ of $\mathscr{A}$ in which a Heisenberg dynamics can be defined, but whose folia are not physical, whereas the opposite is not possible [6].

In Sect. IV it is demonstrated that the Schrödinger dynamics is given directly by $\varphi_{t}^{Q}$ on $E$ for all states in the (time invariant) set $S^{p}(\mathscr{A})$. This result is intimately connected with those of [13]; see also [16] for related work. The precise description of $\left.v_{t}^{Q}\right|_{S^{p}}$ gives rise (Sect. V) to a method of constructing physical folia explicitly: to every $\varphi_{t}^{Q}$-invariant set of probability measures on $E$ one can naturally associate a physical folium whose associated phasespace is given by the supports of the measures. To illustrate our theory, we conclude the paper by considering an explicit physical example, the BCS-model (Sect. VI); in particular, the set $\mathbb{F}_{\text {phys }}$ is there seen to be of amazing richness.

\section{Dynamical Descirptions of a Class of Mean-Field Quantum Systems}

In this preliminary section, we briefly sketch the theory of Bona [10] to the extent necessary for the present purposes, and some structures developed in [12]. For 
more information and additional references, we refer the reader to the original papers. General background on the operator algebraic techniques which we employ throughout can be found in $[17,18]$, and on the properties of folia in [5].

The models under consideration are defined by three elements:

1. The quasilocal $C^{*}$-algebra

$$
\mathscr{A}:=\bigotimes_{n \in \mathbb{N}} \mathscr{A}_{n}, \quad \mathscr{A}_{n}=B\left(\mathbb{C}^{m}\right)=M_{m} \forall n \in \mathbb{N}
$$

where $M_{m}$ are the complex $m \times m$-matrices. $\mathscr{A}$ is simple, so all folia $F \subseteq S(\mathscr{A})$ are full, and all states $S(\mathscr{A})$ are locally normal, since the local algebras

$$
\mathscr{A}(\Lambda):=\bigotimes_{n \in \Lambda} \mathscr{A}_{n}, \quad \Lambda \in \mathscr{L}:=\{\Lambda \subseteq \mathbb{N},|\Lambda|<\infty\}
$$

$(|\Lambda|$ denotes the number of elements in $\Lambda)$ are finite dimensional.

2. A real Lie-algebra $\mathscr{G}$ of dimension $L$ (taken to be the Lie-algebra of a compact Lie-group $G), \mathscr{G} \subseteq \operatorname{Lie}(S U(m))$, which is represented on $\mathbb{C}^{m}$ by antihermitian operators

$$
\frac{1}{i} X(\beta), \quad \text { where } X(\beta) \in\left(M_{m}\right)_{\text {s.a. }} \forall \beta \in \mathscr{G} \text {. }
$$

This Lie-algebra is chosen to give all elements of $\left(M_{m}\right)_{\text {s.a. }}$ corresponding to physically important observables and in any case all those which occur in the local Hamiltonians below.

3. Local Hamiltonians

$$
H^{\Lambda}:=|\Lambda| Q\left(X_{\Lambda}\left(\beta^{1}\right), \ldots, X_{\Lambda}\left(\beta^{L}\right)\right),
$$

where $Q: \mathbb{R}^{L} \rightarrow \mathbb{R}$ is an arbitrary, nonlinear polynomial. The density operators

$$
\begin{aligned}
& X_{\Lambda}\left(\beta^{k}\right):=\frac{1}{|\Lambda|} \sum_{n \in \Lambda} X_{n}\left(\beta^{k}\right), \quad k=1, \ldots, L, \\
& X_{n}\left(\beta^{k}\right)=1 \otimes \cdots \otimes 1 \otimes \underset{\substack{X \\
\text { position } n}}{X} \otimes 1 \otimes \cdots \otimes 1 \in \mathscr{A}(\Lambda)
\end{aligned}
$$

are defined by means of the elements

$$
X\left(\beta^{k}\right) \in\left(M_{m}\right)_{\text {s.a. }}
$$

here, $\beta^{1}, \ldots, \beta^{L}$ is a basis of the vectorspace $\mathscr{G} \cong \mathbb{R}^{L}$ such that $\operatorname{tr}\left(X\left(\beta^{j}\right) X\left(\beta^{k}\right)\right)=$ const $\cdot \delta^{j k}$.

A "dynamical description" of the system in the Heisenberg picture is then a representation $\pi$ of $\mathscr{A}$ in which the local time evolutions

$$
\tau_{t}^{\Lambda}(x)=e^{i t H^{\wedge}} x e^{-i t H^{\wedge}}, \quad x \in \mathscr{A}
$$

converge to a ${ }^{*}$-automorphism group of (a subalgebra of) $\mathscr{M}_{\pi}:=\pi(\mathscr{A})$ " in the infinite volume limit $\Lambda \rightarrow \infty$. This convergence is understood to hold pointwise in the strong operator topology on $\mathscr{H}_{\pi}$, the representation Hilbertspace of $\pi$; we 
denote such infinite volume limits over the net $\mathscr{L}$ in the strong operator topology by stop $-\lim$. For the representations $\pi$ considered we confine ourselves here to those quasicontained in $\pi_{p}$ (i.e. $\pi \leqq \pi_{p}$ ), the representation belonging to $F^{p}$. It is shown in Appendix 1 that all of Bona's results, which are obtained in the framework of the larger folium

$$
F_{\mathscr{G}}:=\left\{\omega \in S(\mathscr{A}): \operatorname{stop}-\lim _{\Lambda} \pi_{\omega}\left(X_{\Lambda}(\beta)\right)=\operatorname{ex} . \forall \beta \in \mathscr{G}\right\}
$$

remain valid for $F^{p}, \pi_{p}$.

The kinematical structure in which the dynamical theory is defined is thus

$$
\begin{gathered}
(\mathscr{A} \cong) \pi_{p}(\mathscr{A}) \cong \pi_{p}(\mathscr{A})^{\prime \prime}=: \mathscr{M}_{\pi_{p}} \cong \mathscr{B}\left(\mathscr{H}_{\pi_{p}}\right), \\
\mathscr{H}_{\pi_{p}}:=\bigoplus_{\omega \in F^{p}} \mathscr{H}_{\omega} .
\end{gathered}
$$

In this setting we define $\left(\mathscr{Z}_{\pi_{p}}\right.$ denotes the center of $\left.\mathscr{M}_{\pi_{p}}\right)$ :

$$
\begin{aligned}
X_{\pi_{p}}(\beta) & :=\operatorname{stop}-\lim _{\Lambda} \pi_{p}\left(X_{\Lambda}(\beta)\right) \in \mathscr{Z}_{\pi_{p}}, \\
\mathscr{N}_{\mathscr{g}} & :=C^{*}-\text { alg. hull }\left\{X_{\pi_{p}}(\beta), \beta \in \mathscr{G}\right\} \cong \mathscr{Z}_{\pi_{p}} .
\end{aligned}
$$

The limiting operators $X_{\pi_{p}}(\beta)$ are per definition averaged over all local sites $n \in \mathbb{N}$ and thus describe the global, "macroscopic" behavior of the system. They can therefore be called "macro-observables." The algebra $\mathscr{N}_{\mathscr{G}}$, the smallest $C^{*}$-algebra containing all physically important macro-observables $X_{\pi_{p}}(\beta), \beta \in \mathscr{G}$, can be looked at as the relevant classical part of the description [8], because of its being part of the center of $\mathscr{M}_{\pi_{p}}$. Due to this macroscopic, classical character, $\mathscr{N}_{\mathscr{G}}$ links the microscopic treatment to phenomenological theory.

Let now $\mathscr{G}^{*} \cong \mathbb{R}^{L}$ denote the linear dual of $\mathscr{G}$, and $F^{1}, \ldots, F^{L}$ the coordinates of elements $F \in \mathscr{G}^{*}$ with respect to the dual basis of the basis $\beta^{1}, \ldots, \beta^{L}$ of $\mathscr{G}$. One can then prove [10]

\section{Theorem 2.1.}

1. $\mathcal{N}_{\mathscr{G}} \cong C\left(E_{\mathscr{G}}\right)$, where $E_{\mathscr{G}} \cong \mathscr{G}^{*} \cong \mathbb{R}^{L}$ is a compact, convex set, and $C\left(E_{\mathscr{G}}\right)$ the continuous functions on $E_{\mathscr{G}}$. The ${ }^{*}$-isomorphism $\mathscr{E}_{\mathscr{G}}: C\left(E_{\mathscr{G}}\right) \rightarrow \mathcal{N}_{\mathscr{G}}$ carries the coordinate functions

$$
\begin{aligned}
f_{k}: E_{\mathscr{G}} & \rightarrow \mathbb{R} \quad k=1, \ldots, L \\
\left(F^{1}, \ldots, F^{L}\right) & \mapsto F^{k}
\end{aligned}
$$

to $X_{\pi_{p}}\left(\beta^{k}\right)$.

2. For $\mathscr{C}_{\mathscr{G}}:=C^{*}$-alg. hull $\left\{\pi_{p}(\mathscr{A}), \mathcal{N}_{\mathscr{G}}\right\} \subseteq \mathscr{M}_{\pi_{p}}$, the following holds:

$$
\mathscr{C}_{\mathscr{G}}=\pi_{p}(\mathscr{A}) \otimes \mathscr{N}_{\mathscr{G}} \cong \mathscr{A} \otimes C\left(E_{\mathscr{G}}\right) .
$$

3. There exists a unique *-automorphism group

$$
\tau_{t}^{Q}: \mathscr{C}_{\mathscr{G}} \rightarrow \mathscr{C}_{\mathscr{G}}
$$


such that for "sufficiently small" $t \in \mathbb{R}$ :

$$
\begin{aligned}
\tau_{t}^{Q}(x) & =\operatorname{stop}-\lim _{\Lambda^{\prime}} \pi_{p}\left(\tau_{t}^{\Lambda^{\prime}}(x)\right) \quad \forall x \in \mathscr{A}(\Lambda), \quad \forall \Lambda \in \mathscr{L}, \\
\tau_{t}^{Q}\left(X_{\pi_{p}}(\beta)\right) & =\operatorname{stop}-\lim _{\Lambda} \pi_{p}\left(\tau_{t}^{\Lambda}\left(X_{\Lambda}(\beta)\right)\right) \quad \forall \beta \in \mathscr{G} .
\end{aligned}
$$

Furthermore, $\tau_{t}^{Q}$ leaves the algebra $\mathscr{N}_{\mathscr{g}}$ invariant, but not the algebra $\pi_{p}(\mathscr{A})$.

The *-automorphism group $\tau_{t}^{Q}$ is the limiting Heisenberg dynamics of the infinite system. The set $E_{\mathscr{G}}$ defines, due to the Gelfand representation theorem, the pure states on $\mathscr{N}_{\mathscr{G}}$, the algebra of classical observables, and can therefore be called the "classical phase-space" of the system: a point $F \in E_{\mathscr{G}}$ corresponds to certain well-defined values of the $L$ classical observables $X_{\pi_{p}}\left(\beta^{1}\right), \ldots, X_{\pi_{p}}\left(\beta^{L}\right)$, hence to a set of $L$ macroscopic parameters of the system.

With the help of $E_{\mathscr{g}}$, the "classical part" of the dynamics, $\left.\tau_{t}^{Q}\right|_{\mathcal{N}_{g},}$, can be described as follows: The polynomial $Q: \mathbb{R}^{L} \rightarrow \mathbb{R}$ can be considered as an element of $C^{\infty}\left(\mathscr{G}^{*}\right)$, since $\mathscr{G} \cong \mathscr{G}^{*} \cong \mathscr{G}^{* *} \cong \mathbb{R}^{L}$ as vector spaces. It induces canonically (i.e. by means of the Lie bracket $\left[\cdot^{\cdot}\right]$ on $\mathscr{G},\left[\beta^{i}, \beta^{j}\right]=\sum_{k=1}^{L} C_{k}^{i j} \beta^{k}$; see [10] for details) a flow $\varphi_{t}^{Q}$ on $E_{\mathscr{G}} \subseteq \mathscr{G}^{*}$ which is generated by the vectorfield $\lambda_{Q}: \mathbb{R}^{L} \rightarrow \mathbb{R}^{L}$ given by

$$
\left(\lambda_{Q}\left(F^{1}, \ldots, F^{L}\right)\right)^{k}=\sum_{j=1}^{L} \frac{\partial Q}{\partial F_{j}}\left(F^{1}, \ldots, F^{L}\right)\left(\sum_{l=1}^{L} C_{l}^{k j} F^{l}\right) .
$$

Thus we can define

$$
\begin{aligned}
\left(\varphi_{t}^{Q}\right)^{*}: C\left(E_{\mathscr{G}}\right) & \rightarrow C\left(E_{\mathscr{G}}\right) \\
f & \mapsto f \circ\left(\varphi_{t}^{Q}\right)
\end{aligned}
$$

as a *automorphism group of $C\left(E_{\mathscr{G}}\right)$. The following result is proven in [10]:

Theorem 2.2. $\left.\tau_{t}^{Q}\right|_{\mathcal{N}_{\mathscr{G}}}=\mathscr{E}_{\mathscr{G}}{ }^{\circ}\left(\varphi_{t}^{Q}\right)^{*} \circ \mathscr{E}_{\mathscr{G}}^{-1}$.

Theorem 2.2 means that one can translate the algebraic description of the classical part of the time evolution into the familiar form of a flow on a finite dimensional coordinate space (where the coordinates signify the macroscopic parameters of the system), given by a set of ordinary (nonlinear) differential equations. In particular, it is clear that we have here a rigorous "contraction of description": the evolution of macroscopic quantities is selfdetermined.

The dynamical description established so far is completed by the results of [13]. There it is shown that $\tau_{t}^{Q}$ can be extended uniquely to a group of $W^{*}$-automorphisms of $\mathscr{C}_{\mathscr{G}}^{\prime \prime}=\mathscr{M}_{\pi_{p}}$, which we denote by the same symbol $\tau_{t}^{Q}$, and that (1a), (1b) hold for all times. This Heisenberg dynamics $\tau_{t}^{Q}: \mathscr{M}_{\pi_{p}} \rightarrow \mathscr{M}_{\pi_{p}}$ induces a predual group $\left(\tau_{t}^{Q}\right)_{*}:\left(\mathscr{M}_{\pi_{p}}\right)_{*} \rightarrow\left(\mathscr{M}_{\pi_{p}}\right)_{*}$ and thus, because of the one-to-one correspondence between normal states on $\mathscr{M}_{\pi_{p}}$ and $F^{p}$, a group

$$
v_{t}^{Q}: F^{p} \rightarrow F^{p}
$$

of affine, normcontinuous bijections of $F^{p}$. The $v_{t}^{Q}$ are obviously to be regarded 
as the time translations in the Schrödinger picture and fulfill (by construction)

$$
v_{t}^{Q}(\omega)(x)=\lim _{\Lambda} \omega\left(\tau_{t}^{\Lambda}(x)\right), \quad \forall \omega \in F^{p}, \quad \forall t \in \mathbb{R}, \quad \forall x \in \mathscr{A}(\Lambda) ; \quad \forall \Lambda \in \mathscr{L} .
$$

Thus, according to the definition in [3], $F^{p}$ is a physical folium, and in the framework of $F^{p}, \pi_{p}$ we have both the Heisenberg picture and the Schrödinger picture, related to each other by duality as usual.

If we now look for possible dynamical subdescriptions of the system, there are two questions:

1. Which representations $\pi \leqq \pi_{p}$ are such that the local time evolutions $\tau_{t}^{\Lambda}$ converge to a ${ }^{*}$-automorphism group in $\mathscr{M}_{\pi}$, i.e. in which $\pi \leqq \pi_{p}$ can a Heisenberg dynamics be defined?

2. Which folia are invariant under $v_{t}^{Q}$, i.e. in which $F \cong F^{p}$ can a Schrödinger dynamics be defined?

To answer these questions, we introduce the following structures: Every $\omega \in F^{p}$ can be considered a normal state on $\mathscr{M}_{\pi_{p}}$, thus by restriction a state on $\mathscr{N}_{\mathscr{g}}$; this state induces via the ${ }^{*}$-isomorphism $\mathscr{E}_{\mathscr{G}}$ a state on $C\left(E_{\mathscr{G}}\right)$, denoted by $\mu_{\omega}$, hence a probability measure on $E_{\mathscr{G}}$, again denoted by $\mu_{\omega}$. The support of $\mu_{\omega}$, $\operatorname{supp} \mu_{\omega}$, is then a closed subset of $E_{\mathscr{G}}$.

If one defines

$$
\mathbb{F}:=\left\{F \cong F^{p}: F \text { folium }\right\}, \text { the set of all subfolia of } F^{p},
$$

-with the lattice operations: $F_{1} \vee F_{2}:=$ smallest folium containing $F_{1}$ and $F_{2}$, $F_{1} \wedge F_{2}:=F_{1} \cap F_{2}$, this is a complete Boolean lattice-and

$$
\mathbb{E}_{\mathscr{G}}:=\left\{B \cong E_{\mathscr{G}}: B \text { closed }\right\}, \quad \text { the set of all closed subsets of } E_{\mathscr{G}},
$$

- this is a complete distributive lattice - it can be proven [12]:

Theorem 2.3. The map

$$
\begin{aligned}
j_{\mathscr{G}}: \mathbb{F} & \rightarrow \mathbb{E}_{\mathscr{G}} \\
F & \mapsto \bigcup_{\omega \in \boldsymbol{F}} \operatorname{supp} \mu_{\omega}
\end{aligned}
$$

is surjective, order-preserving and a complete $\vee$-homomorphism, i.e.

$$
j_{\mathscr{G}}\left(\underset{i \in I}{\vee} F_{i}\right)=\bigvee_{i \in I}^{\vee} j_{\mathscr{G}}\left(F_{i}\right)
$$

for an arbitrary family $\left\{F_{i}: i \in I\right\} \cong \mathbb{F}$ of folia.

Furthermore, every closed set $B \cong E_{\mathscr{G}}$ has a unique maximal preimage: the set $F_{B}:=\left\{\omega \in F^{p}: \operatorname{supp} \mu_{\omega} \cong B\right\}$ is a folium with the property $F \cong F^{p}, j_{\mathscr{G}}(F)=B \Rightarrow F \cong F_{B}$.

The physical meaning of the map $j_{\mathscr{G}}$ is the following. $\mu_{\omega}$ is the classical probability distribution of the macroscopic parameters in the (ensemble of) system(s) described by $\omega \in F^{p}$. Hence, $j_{\mathscr{G}}$ associates to a set of states $F \in \mathbb{F}$ the range of values of the macroscopic variables in which a system belonging to an ensemble 
described by any $\omega \in F$ may be found. In light of this, we call $j_{\mathscr{G}}(F) \subseteq E$ the "classical statespace" associated to the folium $F$.

The map $j_{\mathscr{G}}$ allows a rather straightforward answer to the first question posed above. If $F_{\pi}$ denotes the folium associated to a representation $\pi$, one has [12]

Theorem 2.4. Let $\pi \leqq \pi_{p}$ such that $\varphi_{t}^{Q}\left(E_{\pi}^{\mathscr{G}}\right)=E_{\pi}^{\mathscr{G}}, E_{\pi}^{\mathscr{G}}:=j_{\mathscr{G}}\left(F_{\pi}\right)$. Then a Heisenberg dynamics exists in $\pi$ and it can be defined as $a^{*}$-automorphism group $\tau_{t}^{Q}$ of $\pi(\mathscr{A}) \otimes \mathscr{E}_{\mathscr{G}}\left(C\left(E_{\pi}^{\mathscr{G}}\right)\right)$

Thus, the $\varphi_{t}^{Q}$-invariance of $j_{\mathscr{G}}\left(F_{\pi}\right)$ is sufficient for the existence of a Heisenberg dynamics in $\pi$.

[Remark. There is a converse of Theorem 2.4 in the sense that the $\varphi_{t}^{Q}$-invariance of $E_{\pi}^{\mathscr{G}}$ is also necessary for the local time evolutions $\tau_{t}^{\Lambda}$ to converge to a *-automorphism group on $\pi(\mathscr{A}) \otimes \mathscr{E}_{\mathscr{G}}\left(C\left(E_{\pi}^{\mathscr{G}}\right)\right)$ [12].]

Theorem 2.4 shows that dynamical subdescriptions of the system in the Heisenberg picture can be constructed by determining just the invariant sets of the classical part of the dynamics, and hence are quite easily accessible.

Unfortunately, the situation is much more difficult in the Schrödinger picture; however, the flow $\varphi_{t}^{Q}$ does contain a lot of information about $v_{t}^{Q}$, as we shall see in the next sections, and thus one can give at least a partial answer to question 2 as well.

To conclude this section, we remark that everything that has been stated so far remains true if one replaces the Lie-algebra $\mathscr{G}$ by any bigger Lie-algebra $\mathscr{G}^{\prime}$, $\mathscr{G} \subseteq \mathscr{G}^{\prime} \subseteq \operatorname{Lie}(S U(m))$. Thus, we shall henceforth suppress the index $\mathscr{G}$.

\section{The Schrödinger Dynamics $v_{t}^{Q}$}

We agree with the viewpoint implicitly taken in $[6,7]$ that one should require the existence of both the Heisenberg picture and the Schrödinger picture for a physically adequate description of the system, since the time development of states is an integral part of quantum theory.

The following theorem shows that looking for such descriptions is in fact equivalent to question 2 above:

Theorem 3.1. For a representation $\pi \leqq \pi_{p}$ the following conditions are equivalent:

1. A limiting Heisenberg dynamics $\tau_{t}^{Q}$ exists in $\pi$, is $\sigma\left(\mathscr{M}_{\pi},\left(\mathscr{M}_{\pi}\right)_{*}\right)$-continuous and thus allows a Schrödinger picture as the predual $\left(\tau_{t}^{Q}\right)_{*}:\left(\mathscr{M}_{\pi}\right)_{*,+, 1} \rightarrow\left(\mathscr{M}_{\pi}\right)_{*,+, 1}$.

2. $\tau_{t}^{Q}(c(\pi))=c(\pi)$, where $c(\pi) \in \mathscr{M}_{\pi_{p}}$ is the central projection corresponding to $\pi$.

3. $v_{t}^{Q}\left(F_{\pi}\right)=F_{\pi}$.

4. $F_{\pi}$ is a physical folium of the system.

Proof. (1) $\Leftrightarrow(2) \Leftrightarrow(3)$ is well known, (3) $\Leftrightarrow(4)$ follows from Eq. (2).

The physically adequate subdescriptions of the system described by $\left(\mathscr{M}_{\pi_{p}}, F^{p}, \tau_{t}^{Q}\right.$ : $\mathscr{M}_{\pi_{p}} \rightarrow \mathscr{M}_{\pi_{p}}$ ) are thus given by the set

of physical folia.

$$
\mathbb{F}_{\text {phys }}:=\left\{F \in \mathbb{F}: v_{t}^{Q}(F)=F\right\}
$$


As discussed in the introduction, the aim of this paper is to obtain more information about the Schrödinger dynamics $v_{t}^{Q}$ and thereby about $\mathbb{F}_{\text {phys }}$. The global structure of these objects is contained in

\section{Theorem 3.2.}

1. $v_{t}^{Q}$ is a group of complete lattice automorphisms of $\mathbb{F}$, i.e.

$$
\begin{aligned}
& v_{t}^{Q}\left(\bigvee_{i \in I}^{\vee} F_{i}\right)=\bigvee_{i \in I}^{\vee} v_{t}^{Q}\left(F_{i}\right), \\
& v_{t}^{Q}\left(\bigwedge_{i \in I}^{\wedge} F_{i}\right)=\bigwedge_{i \in I} v_{t}^{Q}\left(F_{i}\right)
\end{aligned}
$$

for an arbitrary family $\left\{F_{i}: i \in I\right\}$ of folia.

In particular, $v_{t}^{Q}$ maps folia onto folia.

2. $\mathbb{F}_{\text {phys }}$ is a complete Boolean lattice.

Proof. (1) This result is known, but can hardly be found in the literature in this formulation (compare [9]). We therefore sketch the proof. Since $\mathbb{F}$ is isomorphic as a complete Boolean lattice to the lattice of central projections $C P\left(\mathscr{M}_{\pi_{p}}\right)$ in $\mathscr{M}_{\pi_{p}}$ and $c\left(v_{t}^{Q}(F)\right)=\tau_{t}^{Q}(c(F)) \forall F \cong F^{p}$, the theorem can be proved equivalently for $\tau_{t}^{Q}$ on $C P\left(\mathscr{M}_{\pi_{p}}\right)$. Here we have

$$
\begin{aligned}
& c_{1} \vee c_{2}=c_{1}+c_{2}-c_{1} c_{2}, \\
& c_{1} \wedge c_{2}=c_{1} c_{2},
\end{aligned}
$$

so that the lattice-automorphism property of $\tau_{t}^{Q}$ is trivial. The completeness of this automorphism follows from the fact that

$$
\underset{i \in I}{\vee} c_{i}=\sigma\left(\mathscr{M}_{\pi_{p}},\left(\mathscr{M}_{\pi_{p}}\right)_{*}\right)-\underset{\Lambda}{\lim } \vee c_{i \in \Lambda}
$$

where the limit is performed over the net $\{\Lambda \subseteq I:|\Lambda|<\infty\}$, and from the $\sigma\left(\mathscr{M}_{\pi_{p}},\left(\mathscr{M}_{\pi_{p}}\right)_{*}\right)-\sigma\left(\mathscr{M}_{\pi_{p}},\left(\mathscr{M}_{\pi_{p}}\right)_{*}\right)$-continuity of $\tau_{t}^{Q}$.

(2) is a trivial consequence of (1).

We now look at the Schrödinger dynamics $v_{t}^{Q}$ more closely; in particular, we are interested in the connection of this quantum state evolution to the time evolution on the macroscopic level, i.e. of the classical states $F \in E$. Indeed, the following theorem, the main result of this section, shows how the action of $v_{t}^{Q}$ on folia is mirrored-or, in other words, constrained-by the flow $\varphi_{t}^{Q}$ on E:

Theorem 3.3. Let $F \subseteq F^{p}$. Then

$$
j\left(v_{t}^{Q}(F)\right)=\varphi_{t}^{Q}(j(F)) .
$$

Proof. We prove first $\mu_{v_{t}^{Q}(\omega)}=\mu_{\omega}^{\circ}\left(\varphi_{t}^{Q}\right)^{-1}$. For all $f \in C(E)$,

$$
\begin{aligned}
\mu_{v_{t}^{Q}(\omega)}(f) & =v_{t}^{Q}(\omega)(\mathscr{E}(f))=\omega\left(\tau_{t}^{Q}(\mathscr{E}(f))\right) \\
& =\omega\left(\mathscr{E}\left(\left(\varphi_{t}^{Q}\right)^{*}(f)\right)\right)=\mu_{\omega}\left(f \circ \varphi_{t}^{Q}\right)
\end{aligned}
$$




$$
\begin{aligned}
& =\int_{E} f \circ \varphi_{t}^{Q} d \mu_{\omega}=\int_{E} f d\left(\mu_{\omega} \circ\left(\varphi_{t}^{Q}\right)^{-1}\right) \\
& =\left(\mu_{\omega}^{\circ}\left(\varphi_{t}^{Q}\right)^{-1}\right)(f) .
\end{aligned}
$$

This entails supp $\mu_{v_{t}^{Q}(\omega)}=\varphi_{t}^{Q}\left(\operatorname{supp} \mu_{\omega}\right)$, since $\varphi_{t}^{Q}$ is continuous. We then have

$$
\begin{aligned}
j\left(v_{t}^{Q}(F)\right) & =\overline{\bigcup_{\omega \in v_{t}^{Q}(F)} \operatorname{supp} \mu_{\omega}}=\overline{\bigcup_{\omega \in F} \operatorname{supp} \mu_{v_{t}^{Q}(\omega)}} \\
& =\overline{\bigcup_{\omega \in F} \varphi_{t}^{Q}\left(\operatorname{supp} \mu_{\omega}\right)}=\varphi_{t}^{Q}\left(\overline{\bigcup_{\omega \in F} \operatorname{supp} \mu_{\omega}}\right) \\
& =\varphi_{t}^{Q}(j(F)) .
\end{aligned}
$$

The picture of the Schrödinger dynamics $v_{t}^{Q}$ given by the flow $\varphi_{t}^{Q}$ is only a "coarse grained" one, since there are in general many folia which are mapped onto the same $B \subseteq E$ by $j$. This is also clear from a physical point of view: the information about the global behavior of the system contained in $\varphi_{t}^{Q}$ is not sufficient to know the details of its behavior on the microscopic level.

Corollary 3.4. Let $F \subseteq F^{p}$. Then

$$
v_{t}^{Q}(F)=F \Rightarrow \varphi_{t}^{Q}(j(F))=j(F) .
$$

In light of Corollary 3.4, the set $\mathbb{F}_{\text {phys }}$ is naturally classified in terms of the "possible sub-phasespaces" $B \subseteq E$, i.e. the closed, $\varphi_{t}^{Q}$-invariant subsets $B$ of $E$ : the set

$$
\mathbb{F}_{\text {phys }}(B):=\left\{F \in \mathbb{F}_{\text {phys }}: j(F)=B\right\} \subseteq \mathbb{F}_{\text {phys }}
$$

gives all possible dynamical descriptions of the system with one and the same associated classical statespace $B \subseteq E$, i.e. with the same "macroscopic structure." As pointed out in the introduction, $B$ corresponds physically to specified ranges of values of the macroscopic observables, to which the system may be confined due to external circumstances.

Lemma 3.5. Let $B \subseteq E$ be a phasespace. $\mathbb{F}_{\mathrm{phys}}(B)$ is not empty and contains a unique maximal element, $F_{B}$.

Proof. Since $j\left(v_{t}^{Q}\left(F_{B}\right)\right)=B$, we have by maximality of $F_{B}: v_{t}^{Q}\left(F_{B}\right) \subseteq F_{B}$. The group property of $v_{t}^{Q}$ then yields $v_{t}^{Q}\left(F_{B}\right)=F_{B}$.

For every external physical situation, one can thus choose a dynamical description which is maximal in the sense that it contains all states (in the framework of $F^{p}$ ) in which the system can be under these circumstances. We shall show in Sect. $\mathrm{V}$ that, in general, $\mathbb{F}_{\text {phys }}(B)$ in fact contains many (even disjoint) elements.

We conclude this section with a remark concerning Heisenberg picture and Schrödinger picture of the dynamics. It is clear from Corollary 3.4 that $\varphi_{t}^{Q}$ invariance of $j(F)$ is a necessary condition for $F$ to be a physical folium. It is, however, not sufficient: 
Proposition 3.6. Let $B \subseteq E$ be a phasespace, consisting not only of invariant points. Then there is a $F \in \mathbb{F}$ such that

(i) $\varphi_{t}^{Q}(j(F))=j(F)$.

(ii) $v_{t}^{Q}(F) \neq F$.

\section{Proof. See Appendix 2.}

Recalling Theorem 2.4 , it is now clear that there are representations $\pi$ of $\mathscr{A}$ in which a Heisenberg dynamics exists (as a ${ }^{*}$-automorphism group $\tau_{t}^{Q}$ of $\left.\pi(\mathscr{A}) \otimes \mathscr{E}\left(C\left(E_{\pi}\right)\right)\right)$ which do not allow a Schrödinger picture, i.e. where $\tau_{t}^{Q}$ is not extendable to a $W^{*}$-automorphism group of $\mathscr{M}_{\pi}$. This is to be contrasted with the fact that every physical folium $F$ induces a Heisenberg dynamics in the representation $\pi_{F}$ (Theorem 3.1; compare [6]; the same conclusion could here also be drawn from Theorem 2.4 together with Corollary 3.4). While, mathematically, the Heisenberg picture is thus the more general approach to dynamical descriptions of infinite mean-field quantum systems, we think that the requirement stated at the beginning of the section is adequate from a physical point of view.

\section{The Schrödinger Dynamics $v_{t}^{Q}$ on $S^{p}(\mathscr{A})$}

An even more intimate relation between $v_{t}^{Q}$ and $\varphi_{t}^{Q}$ than given in Theorem 3.3 is revealed if we consider the action of $v_{t}^{Q}$ on the set of $S^{p}(\mathscr{A}) \subseteq F^{p}$ of the permutation invariant states on $\mathscr{A}$.

For clarity of exposition, we shall from now on consider only the most general structures, i.e. those in the case $\mathscr{G}=\operatorname{Lie}(S U(m))$. Relevant substructures, i.e. those belonging to subalgebras $\mathscr{G} \subseteq \operatorname{Lie}(S U(m))$, will be treated in Appendix 3. $S^{p}(\mathscr{A})$ is $v_{t}^{Q}$-invariant due to the permutation invariance of the local Hamiltonians.

Let us first note some facts about $S^{p}$ and its extremal boundary, $\partial S^{p}$ :

\section{Lemma 4.1}

(i) $\varphi \in \partial S^{p} \Rightarrow \varphi=\underset{n \in \mathbb{N}}{\otimes} \rho, \rho \in S\left(M_{m}\right)$.

(ii) $\varphi \in \partial S^{p} \Rightarrow \varphi$ is a factor state.

(iii) $\varphi, \varphi^{\prime} \in \partial S^{p}, \varphi \neq \varphi^{\prime} \Rightarrow \varphi, \varphi^{\prime}$ disjoint.

(iv) $\omega \in S^{p} \Rightarrow \exists$ a unique measure $\rho_{\omega}$, concentrated on $\partial S^{p}$, such that

$$
\omega(x)=\int_{\partial S^{p}} \varphi(x) d \rho_{\omega}(\varphi) \quad \forall x \in \mathscr{A}
$$

This means that $S^{p}$ is a simplex. Since $\partial S^{p}$ is $w^{*}$-compact, it is even a so-called Bauer-simplex.

(v) For $\omega \in S^{p}, \rho_{\omega}$ its measure according to (iv), it holds

$$
\omega(x)=\int_{\partial S^{p}} \varphi(x) d \rho_{\omega}(\varphi) \quad \forall x \in \mathscr{M}_{\pi_{p}} .
$$

Proof. The proof of (i)-(iv) can be found, e.g., in [19]. (v) Kaplanski's density theorem implies that $\pi(\mathscr{A})_{1}$, the unit ball in $\pi(\mathscr{A})$, is $\sigma\left(\mathscr{M}_{\pi_{p}},\left(\mathscr{M}_{\pi_{p}}\right)_{*}\right)$-dense in $\left(\mathscr{M}_{\pi_{p}}\right)_{1}$. Therefore, for any $x \in \mathscr{M}_{\pi_{p}}$ there exists a sequence $\left\{x_{n}\right\}_{n \in \mathbb{N}} \cong \pi(\mathscr{A})$, 
$\left\|x_{n}\right\| \leqq\|x\|$, with $x_{n} \rightarrow x$ in the $\sigma\left(\mathscr{M}_{\pi_{p}},\left(\mathscr{M}_{\pi_{p}}\right)_{*}\right)$-topology. Therefore,

$$
\begin{aligned}
\omega(x) & =\lim _{n} \omega\left(x_{n}\right)=\lim _{n} \int_{\partial S^{p}} \varphi\left(x_{n}\right) d \rho_{\omega}(\varphi) \\
& \stackrel{L D C}{=} \int_{\partial S^{p}} \lim \varphi\left(x_{n}\right) d \rho_{\omega}(\varphi) \\
& =\int_{\partial S^{p}} \varphi(x) d \rho_{\omega}(\varphi),
\end{aligned}
$$

where the Lebesgue dominated convergence theorem applies since $\left\|\varphi\left(x_{n}\right)\right\| \leqq$ $\left\|x_{n}\right\| \leqq\|x\| \forall n \in \mathbb{N}$, and $\rho_{\omega}$ is a probability measure. The first and last equalites hold because all $\omega \in S^{p} \subseteq F^{p}=\left(\mathscr{M}_{\pi_{p}}\right)_{*,+, 1}$ are normal.

The part (i) of the Lemma implies that we can parametrize $\partial S^{p}$ by parametrizing $S\left(M_{m}\right)$. The operators

$$
X\left(\beta^{1}\right), \ldots, X\left(\beta^{m^{2}-1}\right), 1
$$

form a selfadjoint basis of $M_{m}$ (orthogonal with respect to the trace-scalarproduct), where $\beta^{1}, \ldots, \beta^{m^{2}-1}$ is the basis of $\mathscr{G}=\operatorname{Lie}(S U(m))$. Any $\rho \in S\left(M_{m}\right)$ is uniquely determined by its expectation values on $X\left(\beta^{1}\right), \ldots, X\left(\beta^{m^{2}-1}\right)$ and thus can be considered a linear functional on $\mathscr{G}$, i.e. an element of $\mathscr{G}^{*}$. The map

$$
\begin{gathered}
\mathscr{P}: \partial S^{p} \rightarrow S\left(M_{m}\right) \rightarrow \mathscr{G}^{*} \cong \mathbb{R}^{m^{2}-1}, \\
\varphi=\underset{n \in \mathbb{N}}{\otimes} \rho \mapsto \rho \mapsto\left(\rho\left(X\left(\beta^{1}\right)\right), \ldots, \rho\left(X\left(\beta^{m^{2}-1}\right)\right)\right)
\end{gathered}
$$

is then a $w^{*}$-norm-continuous bijection [19], implying that $\mathscr{P}\left(\partial S^{p}\right)=: D \leqq \mathbb{R}^{m^{2}-1}$ is a compact, convex set.

This induces a one-to-one correspondence between probability measures on $\partial S^{p}$, i.e. $S^{p}$, and probability measures on $D$, denoted by $M(D)_{+, 1}$ :

$$
\begin{aligned}
\overline{\mathscr{P}}: S^{p} & \rightarrow M(D)_{+, 1}, \\
\omega & \mapsto \rho_{\omega} \circ \mathscr{P}^{-1}=: \overline{\rho_{\omega}}, \\
\varphi \in \partial S^{p} & \mapsto \overline{\rho_{\varphi}}=\delta_{\mathscr{P}(\varphi)} .
\end{aligned}
$$

$\overline{\mathscr{P}}$ is an affine, $w^{*}-\sigma\left(C(D)^{*}, C(D)\right)-$ continuous bijection. These structures are connected intimately to the phasespace $E \subseteq \mathbb{R}^{m^{2}-1}$.

\section{Proposition 4.2.}

(i) For $\varphi \in \partial S^{p}: \mu_{\varphi}=\delta_{\mathscr{P}(\varphi)}=\overline{\rho_{\varphi}}$.

(ii) For $\omega \in S^{p}: \mu_{\omega}=\overline{\rho_{\omega}}$ as measures on $\mathbb{R}^{m^{2}-1}$.

(iii) $E=D \cong S\left(M_{m}\right)$.

Proof. (i) Since $\varphi$ is a factor state, $\mu_{\varphi}$ is a Dirac-measure on $E$ [12], concentrated on a point $F_{\varphi}:=\left(F_{\varphi}^{1}, \ldots, F_{\varphi}^{m^{2}-1}\right) \in E$, i.e. $\mu_{\varphi}=\delta_{F_{\varphi}}$. But $F_{\varphi}=\mathscr{P}(\varphi)$, since

$$
F_{\varphi}^{k}=f_{k}\left(F_{\varphi}\right)=\mu_{\varphi}\left(\left(f_{k}\right)\right)=\varphi\left(X_{\pi_{p}}\left(\beta^{k}\right)\right) \stackrel{!}{=} \rho\left(X\left(\beta^{k}\right)\right)=(\mathscr{P}(\varphi))^{k}
$$


for all $k=1, \ldots, m^{2}-1$, where (!) can be verified by direct calculation. The relation $\mu_{\varphi}=\delta_{\mathscr{P}(\varphi)}$ also implies $D \subseteq E$, since $D=\bigcup_{\varphi \in \partial S^{p}} \mathscr{P}(\varphi)=\bigcup_{\varphi \in \partial S^{p}} \operatorname{supp} \mu_{\varphi} \subseteq E$. (Relation

(i) was also proven in [11]).

(ii) Due to Lemma 4.1(v), one has for all $f \in C(E)$ :

but then

$$
\omega(\mathscr{E}(f))=\int_{\partial S^{p}} \varphi(\mathscr{E}(f)) d \rho_{\omega}(\varphi)
$$

$$
\left.\mu_{\omega}(f)=\omega(\mathscr{E}(f))=\int_{\partial S^{p}} \varphi(\mathscr{E}(f)) d \rho_{\omega}(\varphi)=\int_{\partial S^{p}} \mu_{\varphi}(f)\right) d \rho_{\omega}(\varphi)
$$

and, due to part (i),

$$
=\int_{\partial S^{p}} f(\mathscr{P}(\varphi)) d \rho_{\omega}(\varphi)=\int_{\mathscr{P}\left(\partial S^{p}\right)} f(F) d\left(\rho_{\omega}^{\circ} \mathscr{P}^{-1}\right)(F)=\int_{D} f(F) d \overline{\rho_{\omega}}(F)
$$

The result $\mu_{\omega}=\overline{\rho_{\omega}}$ follows due to the one-to-one correspondence between states on $C(D)$ and $M(D)_{+, 1}$. In particular, supp $\mu_{\omega} \subseteq D$ for all $\omega \in S^{p}$.

(iii) It was shown in [12] that

$$
j(F)=\overline{\bigcup_{\omega \in F} \operatorname{supp} \mu_{\omega}}=\overline{\bigcup_{\omega \in \mathbb{Q}} \operatorname{supp} \mu_{\omega}},
$$

if $Q$ is a generating set for the folium $F$, i.e. $F=$ convhull $\left\{Q_{c}\right\}^{-\|\cdot\|}, Q_{c}:=$ $\left\{\psi \in S(\mathscr{A}): \exists c \in \mathscr{A}, \omega \in Q\right.$ such that $\left.\psi=\omega_{c}:=\omega\left(c^{*} \cdot c\right) / \omega\left(c^{*} c\right)\right\}$. Since $S^{p}$ is a generating set for $F^{p}$, we get

$$
E=j\left(F^{p}\right)=\bigcup_{\omega \in S^{p}} \operatorname{supp} \mu_{\omega} \subseteq D
$$

due to (ii). Together with (i), this implies $E=D$. (Compare [11])

The structures developed above can be interpreted as follows. The map $\mathscr{P}$ is a parametrization of the factorstates $\partial S^{p}$ with the macroscopic parameters of the system: to each classical state $F \in E$ there exists one and only one state $\varphi_{F} \in \partial S^{p}$, i.e. one and only one state $\varphi$ such that

- the system in the state $\varphi$ shows the macroscopic parameters $F^{1}, \ldots, F^{m^{2}-1}$;

- every local site $n \in \mathbb{N}$ is in the same state (permutation-invariance), i.e. the system is completely homogeneous.

Likewise, the states $\omega \in S^{p}$ are uniquely determined by their "classical part" $\left.\omega\right|_{\mathcal{N}}$ only, since $\rho_{\omega}=\left(\left.\omega\right|_{\mathscr{N}^{\circ}} \mathscr{E}^{-1}\right) \circ \mathscr{P}$; conversely, each measure $\eta$ on $E$ gives a unique $\omega_{\eta} \in S^{p}, \omega_{\eta}=\overline{\mathscr{P}}^{-1}(\eta)$, with $\mu_{\omega_{\eta}}=\eta$. These particular properties of $S^{p}$ imply that the Schrödinger dynamics on $S^{p}$,

$$
\left.v_{t}^{Q}\right|_{S^{p}}: S^{p} \rightarrow S^{p}
$$

is directly given by the classical flow $\varphi_{t}^{Q}$ on $E$ :

\section{Corollary 4.3.}

(i) $\left.v_{t}^{Q}\right|_{\partial S^{p}}=\mathscr{P}^{-1} \circ \varphi_{t}^{Q} \circ \mathscr{P}$. 
(ii) $\left.v_{t}^{Q}\right|_{S^{p}}=\overline{\mathscr{P}}^{-1} \circ\left(\varphi_{t}^{Q}\right)^{* * \circ} \circ \overline{\mathscr{P}}$, where

$$
\begin{aligned}
\left(\varphi_{t}^{Q}\right)^{* *}: M(E)_{+, 1} & \rightarrow M(E)_{+, 1} \\
\eta & \mapsto \eta \circ\left(\varphi_{t}^{Q}\right)^{-1}
\end{aligned}
$$

Proof. (ii) follows from 4.2(ii) and the general relation $\mu_{v_{t}^{Q}(\omega)}=\mu_{\omega}^{\circ}\left(\varphi_{t}^{Q}\right)^{-1}$ proven for Theorem 3.3, (i) is a special case of (ii).

We have here the remarkable fact that the full quantum Schrödinger dynamics of a class of physically distinguished states of an infinite system can be described by a finite set of differential equations. In particular, this result shows that the classical flows $\varphi_{t}^{Q}$, defined here following [10], and the one defined in [13], coincide. Let us also remark that, in case of quadratic nonlinearity of $Q$, the Schrödinger dynamics on $\partial S^{p}$ has been described differently, with a nonlinear Schrödinger equation on $S\left(M_{m}\right)$, in [16]; of course, one can show explicitly that the two treatments are equivalent.

\section{Physical Folia}

In this section, we turn to the notion of a physical folium and show how such folia can be constructed explicitly with help of the results of Sect. IV; in particular, this yields a method of constructing dynamical descriptions of the infinite system in any environmental situation, corresponding to a reduced phasespace $B \subseteq E$. First we need the following

Proposition 5.1. Let $S \subseteq F^{p}$ a set of states with $v_{t}^{Q}(S)=S$, and $F_{S}$ the folium generated by $S$ (the smallest folium containing $S$ ). Then it holds

$$
v_{t}^{Q}\left(F_{S}\right)=F_{S} \text {, }
$$

i.e. $F_{S}$ is a physical folium.

Proof. It is $F_{S}=$ convhull $\left\{S_{c}\right\}^{-\|\cdot\|}$. Since $v_{t}^{Q}$ is affine and norm-continuous, it suffices to show that $v_{t}^{Q}\left(S_{c}\right) \subseteq F_{S}$.

Let $\psi:=\omega_{c} \in S_{c}$ with $\omega \in S, c \in \mathscr{A}$ such that $\omega\left(c^{*} c\right)=1$. One has for all $x \in \pi_{p}(\mathscr{A})$,

$$
\begin{aligned}
v_{t}^{Q}(\psi)(x) & =\psi\left(\tau_{t}^{Q}(x)\right) \\
& =\omega\left(\pi_{p}(c)^{*} \tau_{t}^{Q}(x) \pi_{p}(c)\right) \\
& =\omega\left(\tau_{t}^{Q}\left(\bar{c}^{*} x \bar{c}\right)\right), \quad \bar{c}:=\tau_{-t}^{Q}\left(\pi_{p}(c)\right) \in \mathscr{M}_{\pi_{p}} \\
& =v_{t}^{Q}(\omega)\left(\bar{c}^{*} x \bar{c}\right) \\
& =v_{t}^{Q}(\omega)_{\bar{c}}(x),
\end{aligned}
$$

hence, $v_{t}^{Q}(\psi)=v_{t}^{Q}(\omega)_{\bar{c}}$ on $\mathscr{M}_{\pi_{p}}$ due to normality of these states. $v_{t}^{Q}(\omega)_{\bar{c}}$ is obviously in the folium $\bar{F}_{S}$ of $S\left(\mathscr{M}_{\pi_{p}}\right)$ generated by $S$; but since the folium structure within the normal states on $\mathscr{M}_{\pi_{p}}$ coincides with that within $F^{p}$ ([18], Theorem III.2.7), $\bar{F}_{S}=F_{S}$ and therefore $v_{t}^{Q}(\psi) \in F_{S}$. Since $\psi$ was arbitrary, the result is proved.

The following main result is now easy to see: 
Theorem 5.2. Let $M \subseteq M(E)_{+, 1}$ be a set of measures which is invariant under $\left(\varphi_{t}^{Q}\right)^{* *}$; let $F_{M} \subseteq F^{p}$ be the folium generated by $\overline{\mathscr{P}}^{-1}(M)$. Then $F_{M}$ is invariant under $v_{t}^{Q}$, hence a physical folium.

Proof. Due to Corollary 4.3(ii), $v_{t}^{Q}$ leaves $\overline{\mathscr{P}}^{-1}(M)$ invariant, and thus the theorem follows from Proposition 5.1.

\section{Remarks.}

1. As a special case of Theorem 5.1, invariant states $\omega \in F^{p}$ generate physical folia $F_{\omega}$. In particular, it is known [20] that all limiting Gibbs states of our class of models are time invariant states in $S^{p}$; hence for these models the folia generated by equilibrium states are physical, like it is known for many other cases $[2,4]$.

2. The phasespaces of folia in $\mathbb{F}_{\text {phys }}$ of the type of Theorem 5.2 are explicitly known: we have [12]

$$
j\left(F_{M}\right)=\bigcup_{\eta \in M} \operatorname{supp} \eta \subseteq E .
$$

With this knowledge, it is easy to construct many physical folia in $\mathbb{F}_{\text {phys }}(B)$ for any given $B$. The simplest ones are of course those generated by an invariant state $\omega \in S^{p}(\mathscr{A})$ with supp $\mu_{\omega}=B$.

\section{Examples.}

1. Let $B \subseteq E$ be a phasespace, and $M:=\left\{\delta_{x}: x \in B\right\} \subseteq M(E)_{+, 1}$, where $\delta_{x}$ is the Dirac-measure on $x \in B$. Then

$$
F_{0}(B):=\bigvee_{\varphi \in \mathscr{P}^{-1}(B)} F_{\varphi}
$$

is a physical folium.

2. Let $B \neq\{x\}$ be a minimal phasespace (:= minimal set) for $\varphi_{t}^{Q}$, i.e. every orbit is dense in $B$. Consider any (non-periodic - the following construction can be easily modified for periodic orbits) orbit $\varphi_{\mathbb{R}}^{Q}(x), x \in B$, and any normed measure $\mu$ on $\mathbb{R}$, absolutely continuous with respect to the Lebesgue-measure, with $\operatorname{supp} \mu=\mathbb{R}$. The bijections

$$
\begin{aligned}
\varphi_{t}: \mathbb{R} & \rightarrow \varphi_{\mathbb{R}}^{Q}(x), \\
s & \mapsto \varphi_{s+t}^{Q}(x)
\end{aligned}
$$

give measures $\eta_{t}:=\mu \circ\left(\varphi_{t}^{Q}\right)^{-1}$ on $B$ with supp $\mu=\overline{\varphi_{\mathbb{R}}^{Q}(x)}=B \forall t \in \mathbb{R}$. Obviously, $M:=\left\{\eta_{t}: t \in \mathbb{R}\right\}$ is invariant under $\left(\varphi_{t}^{Q}\right)^{* *}$, hence $F_{M}$ is a physical folium with phasespace $B$. (One can show that $F_{0}(B) \cap F_{M}=\varnothing$.)

\section{Example: The BCS-Model}

In this final section, we consider the BSC-model for a superconductor as a concrete physical example of the theory presented here (compare [11,21]). In this case, $\mathscr{G}=\operatorname{Lie}(S U(2))$, so that $E$ is the state space of the $2 \times 2$-matrices, parametrized by the spin operators $\sigma_{1}, \sigma_{2}, \sigma_{3}$-that is, $E$ is the closed ball with radius $1 / 2$ in $\mathbb{R}^{3}$. 
The local Hamiltonians are (with $X_{\Lambda}^{k}:=\sum_{n \in \Lambda} \sigma_{k}, k=1,2,3$ ):

with

$$
H^{\Lambda}:=|\Lambda| Q\left(X_{\Lambda}^{1}, X_{\Lambda}^{2}, X_{\Lambda}^{3}\right)
$$

$$
Q: \mathbb{R}^{3} \rightarrow \mathbb{R}, \quad Q\left(F^{1}, F^{2}, F^{3}\right)=2 \varepsilon F^{3}-g\left(\left(F^{1}\right)^{2}+\left(F^{2}\right)^{2}\right) .
$$

The induced flow $\varphi_{t}^{Q}$ on $E$ is easily calculable and reads in cylindrical coordinates $z:=F^{3} \quad$ ( $\hat{=}$ particle number), $\quad c:=\left(\left(F^{1}\right)^{2}+\left(F^{2}\right)^{2}\right)^{1 / 2}$ ( $\hat{=}$ number of condensed Cooperpairs), $\varphi:=\arctan \left(F^{2} / F^{1}\right)(\hat{=}$ macroscopic phase):

$$
\varphi_{t}^{Q}(z, c, \varphi)=(z, c, \varphi+(2 \varepsilon+2 g z) t)
$$

it is a rotation around the $z$-axis with $z$-dependent frequency $2 \varepsilon+2 g z$. Physically interesting subphasespaces in this case are, e.g., the spheres $S_{r}, 0 \leqq r \leqq 1 / 2$, composing $E$ : they are precisely the regions of equal temperature, i.e. they represent a situation where the system is in a thermal bath of a temperature $\beta$. This can be seen by looking at the (grandcanonical) equilibrium states of the BCS-model [22].

Dynamical descriptions for such a physical situation, i.e. with phasespace $B=S_{r}, r$ fixed, can be easily constructed from our theory: to every $z_{0} \in[-r, r]$ let $K_{z_{0}}$ denote the circle around the $z$-axis $\left\{z, c, \varphi: z=z_{0}, c=\sqrt{r^{2}-z_{0}^{2}}, \varphi \in[0,2 \pi]\right\} \subseteq S_{r}$. The Lebesgue measure $\lambda\left(K_{z_{0}}\right)$ is obviously invariant under $\varphi_{t}^{Q}$ (in fact, the $v_{t}^{Q}$-invariant state $\overline{\mathscr{P}}^{-1}\left(\lambda\left(K_{z_{0}}\right)\right) \in S^{p}$ is an equilibrium state of the BCS-model). It is then clear that the sets

$$
M_{T}:=\left\{\lambda\left(K_{z}\right): z \in T\right\},
$$

where $T$ is any dense subset of $[-r, r]$, generate physical folia $F_{T}$ with phasespace $S_{r}$. Furthermore, it holds (Lemma 4.1)

$$
F_{T} \cap F_{T^{\prime}}=\varnothing \Leftrightarrow T \cap T^{\prime}=\varnothing .
$$

Hence there are overcountably many disjoint folia in $\mathbb{F}_{\text {phys }}\left(S_{r}\right)$, i.e. overcountably many different dynamical descriptions with the same macroscopic dynamical structure.

As a final comment, we want to emphasize that when we have spoken of a "physically adequate description" of the system, we have not imposed any continuity requirement in the time parameter. The set of all descriptions with strong continuity of $v_{t}^{Q}$ in $t$, or, equivalently, with $\sigma$-weak-continuity of $\tau_{t}^{Q}$ in $t$, will be considered elsewhere.

\section{Appendix 1}

In this appendix, we want to show that the results of [10] continue to hold if one replaces the folium $F_{\mathscr{G}}$ and the representation $\pi_{\mathscr{G}}$ by $F^{p}, \pi_{p}$. From the proofs given there it is clear that all we need to show is that the projection-valued measure $\left(\mathscr{B}\left(\mathscr{G}^{*}\right)\right.$ is the Borel- $\sigma$-algebra of $\left.\mathscr{G}^{*}\right)$

$$
\mathscr{E}_{p}: \mathscr{B}\left(\mathscr{G}^{*}\right) \rightarrow \mathscr{Z}_{\pi_{p}}
$$

constructed from the unitary representation $\mathscr{G} \rightarrow \mathscr{B}\left(\mathscr{H}_{\pi_{p}}\right), \beta \mapsto \exp \left(i X_{\pi_{p}}(\beta)\right)$ with 
help of the SNAG-theorem is $G$-invariant, i.e.

$$
\tilde{\sigma}(g)\left(\mathscr{E}_{p}(B)\right)=\mathscr{E}_{p}\left(\mathrm{Ad}^{*}(g) B\right) \forall B \in \mathscr{B}\left(\mathscr{G}^{*}\right), \quad \forall g \in G .
$$

Here, $\tilde{\sigma}(g)$ denotes the extension to $\mathscr{A}^{* *}$ of the *-automorphism $\sigma(g): \mathscr{A} \rightarrow \mathscr{A}$ defined by

$$
\sigma(g)\left(\bigotimes_{n=1}^{\infty} x_{n}\right)=\bigotimes_{n=1}^{\infty} U(g) x_{n} U(g)^{*}
$$

and linear extension; $U: G \rightarrow M_{m}$ is the unitary representation of the Lie-group $G$ with generators $X(\beta), \beta \in \mathscr{G}$.

To establish (4), it suffices to show [10] that $\tilde{\sigma}(g)\left(c\left(\pi_{p}\right)\right)=c\left(\pi_{p}\right) \forall g \in G$, where $c\left(\pi_{p}\right) \in \mathscr{A}^{* *}$ is the central projection belonging to $\pi_{p}$; or, equivalently, that $\sigma(g)^{*}: \mathscr{A}^{*} \rightarrow \mathscr{A}^{*}$, the dual of $\sigma(g)$, leaves $F^{p}$ invariant. One knows [17] that $\sigma(g)^{*}$ is linear, normpreserving and leaves $S(\mathscr{A}) \subseteq \mathscr{A}^{*}$ invariant. Thus, in order to show $\sigma(g)^{*}\left(F^{p}\right)=F^{p}$ all we need to confirm is $\sigma(g)^{*}\left(S_{c}^{p}\right) \subseteq F^{p}$. This we establish in three steps:

1. $\sigma(g)^{*}\left(\partial S^{p}\right) \subseteq \partial S^{p}: \sigma(g)^{*}(\varphi)=\varphi^{\circ} \sigma(g)=\left(\bigotimes_{n} \rho\right) \circ \sigma(g)=\bigotimes_{n} U(g)^{*} \rho U(g) \in \partial S^{p}$.

2. $\sigma(g)^{*}\left(S^{p}\right) \subseteq S^{p}: \sigma(g)^{*}(\omega)=\omega \circ \sigma(g)=\int \varphi \circ \sigma(g) d \rho_{\omega}(\varphi)=\int \sigma(g)^{*}(\varphi) d \rho_{\omega}(\varphi) \in S^{p}$, due to 1 .

3. $\sigma(g)^{*}\left(S_{c}^{p}\right) \subseteq F^{p}$ :One easily calculates that with $\bar{c}:=\sigma(g)(c) \in \mathscr{A}$ one has $\forall \omega \in S^{p}$, $\forall c \in \mathscr{A}$ :

$$
\sigma(g)^{*}\left(\omega_{c}\right)=\left(\sigma(g)^{*}(\omega)\right)_{\bar{c}} \in S_{c}^{p} .
$$

Thus, $\sigma(g)^{*}\left(S_{c}^{p}\right) \subseteq S_{c}^{p} \subseteq F^{p}$, and hence, $F^{p}$ is invariant under $\sigma(g)^{*}$.

\section{Appendix 2}

Proof of Proposition 3.6. The assumption on $B$ implies that there is a dense set $T \subseteq B$ with $\varphi_{t}^{Q}(T) \neq T$. Consider the folium

We have then

$$
F:=\underset{F \in T}{\bigvee} F_{\varphi_{F}}, \quad \varphi_{F}:=\mathscr{P}^{-1}(F) \in \partial S^{p}(\mathscr{A})
$$

$$
j(F)=\bigvee_{F \in T} j\left(F_{\varphi_{F}}\right)=\bigcup_{F \in T}\{F\}=B .
$$

On the other hand, it holds

$$
v_{t}^{Q}(F)=\bigvee_{F \in T} v_{t}^{Q}\left(F_{\varphi_{F}}\right) \stackrel{(1)}{=} \underset{F \in T}{\vee} v_{t}^{Q}\left(F_{\varphi_{\varphi_{t}^{Q}(F)}}\right)=\underset{F \in \varphi_{t}^{Q}(T)}{\bigvee} F_{\varphi_{F}}^{\stackrel{(i i)}{\neq} F}
$$

Here, (i) follows from Proposition 3.2, (ii) from Lemma 4.1.

\section{Appendix 3}

In this appendix, we extend the relations of Sect. IV to the case where $\mathscr{G}$ is a proper subalgebra of $\operatorname{Lie}(S U(m))$ with basis $\beta^{1}, \ldots, \beta^{L}, L<m^{2}-1$. To choose $\mathscr{G}$ 
smaller than $\operatorname{Lie}(S U(m))$ means that one restricts attention to substructures of all structures belonging to $\operatorname{Lie}(S U(m))$, which are time-invariant due to the fact that the local Hamiltonians are built from the $X(\beta), \beta \in \mathscr{G}$, only. I.e., the algebra of classical observables in this case, $\mathcal{N}_{\mathscr{G}}$, is a $\tau_{t}^{Q}$-invariant subalgebra of $\mathcal{N}(\operatorname{Lie}(S U(m)))$, and $E_{\mathscr{G}}$ is a $\varphi_{t}^{Q}$-invariant subset of $E(\operatorname{Lie}(S U(m)))$. More specifically, if we let

$$
\begin{aligned}
\pi: \mathbb{R}^{m^{2}-1} & \rightarrow \mathbb{R}^{L}, \\
\left(F^{1}, \ldots, F^{m^{2}-1}\right) & \mapsto\left(F^{1}, \ldots, F^{L}\right)
\end{aligned}
$$

be the projection onto the first $L$ coordinates, it holds

\section{Proposition 4.2a}

(i) For $\varphi \in \partial S^{p}: \mu_{\varphi}=\delta_{\pi(\mathscr{P}(\varphi))}$.

(ii) For $\omega \in S^{p}: \mu_{\omega}=\overline{\rho_{\omega}} \circ \pi^{-1}$ as measures on $\mathbb{R}^{L}$.

(iii) $E_{\mathscr{G}}:=\pi(D) \subseteq \mathbb{R}^{L} ; E_{\mathscr{G}}$ is a compact and convex set.

Proof. The proposition is an easy consequence of Proposition 4.2.

(i) $F_{\varphi}=\pi(\mathscr{P}(\varphi))$, since Eq. 3 holds for $k=1, \ldots, L$.

(ii) For all $f \in C\left(E_{\mathscr{G}}\right)$,

$$
\mu_{\omega}(f)=\int_{\partial S^{p}} f(\pi(\mathscr{P}(\varphi))) d \rho_{\omega}(\varphi)=\int_{\pi(D)} f(F) d\left(\overline{\rho_{\omega}} \circ \pi^{-1}\right)(F) .
$$

(iii) From (i) it follows $\pi(D) \subseteq E_{\mathscr{g}}$; (ii) implies supp $\mu_{\omega} \cong \pi(D)$, hence $E_{\mathscr{G}} \leqq \pi(D)$. Compactness and convexity follows from continuity and linearity of $\pi$.

The reason for considering such restrictions is a pragmatic one: if the physically relevant macro-observables are already contained in $\mathcal{N}_{\mathscr{G}}$, it suffices for most purposes to work with the phase space $E_{\mathscr{G}}$ and $\varphi_{t}^{Q}$ on $E_{\mathscr{G}}$, whose dimension $L$ may be considerably smaller than $m^{2}-1$. The price to pay is of course that one is looking only at part of the whole dynamics $\tau_{t}^{Q}$ on $\mathcal{N}(\operatorname{Lie}(S U(m))), \varphi_{t}^{Q}$ on $E(\operatorname{Lie}(S U(m)))$, and thus loses information. This is reflected in the following assertions.

To each probability measure $\eta$ on $E_{\mathscr{G}}$ corresponds now a convex family

$$
S_{\eta}:=\left\{\omega \in S^{p}: \overline{\rho_{\omega}} \circ \pi^{-1}=\eta\right\} \cong S^{p}
$$

of states in $S^{p}$. The Schrödinger dynamics expressed with the flow $\left.\varphi_{t}^{Q}\right|_{E \mathscr{g}}$ on $E_{\mathscr{G}}$ reads:

\section{Corollary 4.3a.}

(i) For $\varphi \in \partial S^{p}: \pi \mathscr{P}\left(v_{t}^{Q}(\varphi)\right)=\left.\varphi_{t}^{Q}\right|_{E s}(\pi \mathscr{P}(\varphi))$,

(ii) $v_{t}^{Q}\left(S_{\eta}\right)=S_{\left(\left(\varphi_{t}^{Q}\right)^{* *(\eta)}\right)}$.

Proof. (i) follows directly from Corollary 4.3(i) and the fact that $\pi\left(\varphi_{t}^{Q}(F)\right)=$ $\left.\varphi_{t}^{Q}\right|_{E, s}(\pi(F))$. (ii) follows from $\mu_{v_{t}^{Q}(\omega)}=\left(\varphi_{t}^{Q}\right)^{* *}\left(\mu_{\omega}\right)$.

Finally, we get in place of Theorem 5.2. 
Theorem 5.2a. Let $\eta$ be a measure on $E_{\mathscr{G}}$ which is invariant under $\left(\varphi_{t}^{Q}\right)^{* *}$. Then the folium generated by $S_{\eta}$ is invariant under $v_{t}^{Q}$, hence a physical folium.

Proof. The proof is analogous to the proof of Theorem 5.2.

Acknowledgements. I would like to thank A. Rieckers for continuous support and encouragement, and all members of the $C^{*}$-group in Tübingen, especially $\mathbf{B}$. Kümmerer, for many helpful discussions. I am indebted to P. Bona for making available to me refs. 10, 11 prior to publication. This work is part of a research project supported by the Deutsche Forschungsgemeinschaft. Financial support of the Studienstiftung des deutschen Volkes is gratefully acknowledged. Finally, I would like to thank the editor for helpful comments.

\section{References}

1. Hepp, K., Lieb, E. H.: Helv. Phys. Acta 46, 573 (1973). In: Batelle Rencontres 1974. Moser, J. K. (ed.) Berlin, Heidelberg, New York: Springer 1975; Hepp, K.: Ann. Phys. (NY) 90, 285 (1975)

2. Dubin, D. A., Sewell, G. L.: J. Math. Phys. 11, 2990 (1970); Emch, G. G., Knops, H. J. F.: J. Math. Phys. 11, 3008 (1970); Jellinek, F.: Commun. Math. Phys. 9, 169 (1968)

3. Sewell, G. L.: Commun. Math. Phys. 33, 43 (1973)

4. It is not possible to cite all relevant works; as examples for early treatments, we mention refs. 2; later works include van Hemmen, L.: Fortschritte Phys. 26, 397 (1978); Roos, H.: Physica 100A, 183 (1980); Duffner, E.: Physica 133A, 187 (1985)

5. Haag, R., Kadison, R. V., Kastler, D.: Commun. Math. Phys. 16, 81 (1970)

6. Sewell, G. L.: Lett. Math. Phys. 6, 209 (1983)

7. Morchio, G., Strocchi, F.: J. Math. Phys. 28, 622 (1987)

8. Rieckers, A.: J. Math. Phys. 25, 2593 (1984)

9. Primas, H.: Chemistry, quantum mechanics and reductionism. Berlin, Heidelberg, New York: Springer 1981

10. Bona, P.: J. Math. Phys. 29, 2223 (1988)

11. Bona, P.: J. Math. Phys. 30, 2994 (1989)

12. Unnerstall, T.: to appear in J. Math. Phys. 31 (1990)

13. Duffner, E., Rieckers, A.: Z. Naturforschung 43, 321 (1988)

14. Kadison, R. V.: Topology 3, [Suppl. 2] 177 (1965)

15. Unnerstall, T.: to appear in the Proceedings of the Conference; Selected Topics in QFT and Mathematical Physics. Liblice June 1989, Singapore: World Scientific,

16. Spohn, H.: Rev. Mod. Phys. 53, 569 (1980)

17. Bratteli, O., Robinson, D. W.: Operator algebras and statistical mechanics I, II. Berlin, Heidelberg, New York: Springer 1979, 1981

18. Takesaki, M.: Theory of operator algebras I. Berlin, Heidelberg, New York: Springer 1979

19. St $\phi$ rmer, E.: J. Funct. Anal. 3, 48 (1969)

20. Fannes, M., Spohn, H., Verbeure, A.: J. Math. Phys. 21, 355 (1980)

21. Thirring, W., Wehrl, A.: Commun. Math. Phys. 4, 303 (1967)

22. Fleig, W.: Acta Phys. Austr. 55, 135 (1983)

Communicated by J. Fröhlich

Received July 11, 1989; in revised form December 4, 1989 
